\title{
Upper Bounds for the Diameter and Height of Graphs of Convex Polyhedra*
}

\section{Gil Kalai}

Institute of Mathematics, Hebrew University of Jerusalem, Jerusalem, Israel, and

IBM Almaden Research Center, San Jose, CA 95120, USA

kalai@,humus.huji.ac.il

\begin{abstract}
Let $\Delta(d, n)$ be the maximum diameter of the graph of a $d$-dimensional polyhedron $P$ with $n$-facets. It was conjectured by Hirsch in 1957 that $\Delta(d, n)$ depends linearly on $n$ and $d$. However, all known upper bounds for $\Delta(d, n)$ were exponential in $d$. We prove a quasi-polynomial bound $\Delta(d, n) \leq n^{2 \log d+3}$.

Let $P$ be a $d$-dimensional polyhedron with $n$ facets, let $\varphi$ be a linear objective function which is bounded on $P$ and let $v$ be a vertex of $P$. We prove that in the graph of $P$ there exists a monotone path leading from $v$ to a vertex with maximal $\varphi$-value whose length is at most $n^{2 \sqrt{n}}$.
\end{abstract}

\section{Introduction}

Let $P$ be a convex polyhedron. The graph of $P$ denoted by $G(P)$ is an abstract graph whose vertices are the extreme points of $P$ and two vertices $u$ and $v$ are adjacent if the interval $[v, u]$ is an extreme edge (=one-dimensional face) of $P$. The diameter of the graph of $P$ is denoted by $\delta(P)$.

Let $\Delta(d, n)$ be the maximum diameter of the graphs of $d$-dimensional polyhedra $P$ with $n$ facets. (A facet is a $(d-1)$-dimensional face.) Thus, $P$ is the set of solutions of $n$ linear inequalities in $d$ variables. It is an old standing problem to determine the behavior of the function $\Delta(d, n)$. The value of $\Delta(d, n)$ is a lower bound for the number of iterations needed for the simplex algorithm for linear programming with any pivot rule. \footnotetext{
C0026.

* This research was supported in part by a BSF grant, by a GIF grant, and by ONR-N00014-91-
} 
In 1957 Hirsch conjectured [2] that $\Delta(d, n) \leq n-d$. Klee and Walkup [9] showed that the Hirsch conjecture is false for unbounded polyhedra. They found examples showing that, for $n \geq 2 d, \Delta(d, n) \geq n-d+\lfloor d / 5\rfloor$. The Hirsch conjecture for convex polytopes ( $=$ bounded polyhedra) is still open. It is also open whether $\Delta(d, n)$ is bounded above by a linear function of $n$ and $d$.

In view of its intrinsic interest and simplicity and its close connections with linear programming, the Hirsch conjecture drew substantial attention over the years. For a recent survey on the Hirsch conjecture and its relatives, see [7].

In 1967 Barnette proved [1], [4] that $\Delta(d, n) \leq n 3^{d-3}$. Barnette's bound is linear in the number of facets but exponential in the dimension. An improved upper bound of a similar asymptotic behavior was found in 1970 by Larman who proved [10] that $\Delta(d, n) \leq n 2^{d-3}$.

We give a quasi-polynomial upper bound for $\Delta(d, n)$. The existence of a polynomial or even a linear upper bound for $\Delta(d, n)$ is still open. In this paper $\log x$ stands for $\log _{2} x$.

\section{Theorem 1.}

$$
\Delta(d, n) \leq n^{2 \log d+3} .
$$

Let $P$ be $d$-dimensional polyhedron with $n$ facets and let $\varphi$ be a linear objective function on $R^{d}$ which is bounded on $P$. A $\varphi$-maximal vertex of $P$ is a vertex of $P$ on which $\varphi$ attains its maximum. A path on the graph of $P$ is called monotone if $\varphi$ is nondecreasing along it. For a vertex $w$ of $P$ let $h(w)$ be the minimum length of a monotone path in $G(P)$ from $w$ to a $\varphi$-maximal vertex of $P$. (A length of a path in $G(P)$ is the number of its edges.) The height of $P$ with respect to $\varphi$, denoted by $h_{\varphi}(P)$, is the maximum of $h(\omega)$ over all vertices $w$ of $P$. (Note: our notion of height is different from the one in [4].) Let $H(d, n)$ denote the maximum value of $h_{\varphi}(P)$ for all $d$-dimensional polyhedra $P$ with $n$ facets, and all linear objective functions $\varphi$ as described above. It is easy to see that $\Delta(d, n) \leq H(d, n)$.

Theorem 2.

$$
H(d, n) \leq n^{2 \sqrt{n}} .
$$

A trivial upper bound on $h_{\varphi}(P)$ is the number vertices of $P$. This gives that $\log H(d, n) \leq c \log \left(\begin{array}{l}n \\ d\end{array}\right)$, for some positive constant $c$. The upper bound (2) gives $\log H(d, n) \leq \sqrt{n} \log n$. This is an improvement on the trivial bound when $n=o\left(d^{2}\right)$.

Let $\mathscr{P}[d, r]$ denote the class of simple $d$-polytopes with the property that every $k$-face has at most $r k$ facets.

Theorem 3. Let $r \geq 2$ be a fixed integer. The diameter of every polytope $P$ in the class $\mathscr{P}[d, r]$ and the height of $P$ with respect to every linear objective function are polynomial ind. The height and the diameter of every polytope in $\mathscr{P}[d, 2]$ is at most $d$. 
Theorem 3 applies to several classes of polyhedra which are of interest in linear programming theory. For example, it gives a polynomial upper bound on the height (and the diameter) of the feasible polyhedra of any generalized flow problem.

The proof of Theorem 1 is given in Section 2. The proofs of Theorems 2 and 3 are given in Section 3. In Section 4 we discuss a general combinatorial context for which our results apply. Section 5 contains speculations on the behavior of the function $\Delta(d, n)$ and on the relation with linear programming.

The results in Section 3 were obtained by the author in November 1990, and were the first subexponential bound for $\Delta(d, n)$. The better bounds of Section 2 were proved in March 1991. Several developments occurred since then. A substantial simplification of the proof of Theorem 1 was obtained by Kleitman (May 1991). His proof is given in a joint research announcement with the author [6]. The author observed (September 1991) how to modify the proofs of the quasipolynomial bounds for $\Delta(d, n)$ to get similar bounds for $H(d, n)$. Finally, the author found (September 1991) a randomized simplex algorithm which takes an expected subexponential $\left(n^{3 \sqrt{d}}\right)$ number of arithmetic operations on every linear programming problem with $d$ variables and $n$ constraints.

\section{Quasi-Polynomial Bounds for the Diameter}

Let $P$ be a $d$-dimensional polyhedron. For a face $F$ of $P$ let $N(F)$ be the set of facets of $P$ with intersect $F$. Clearly, the number of facets of $F$ itself is smaller than $N(F)$.

For two vertices $u$ and $w$ of $P$, define a path of faces between $u$ and $v$ as a sequence of the form $u=v_{1}, F_{1}, v_{2}, F_{2} \ldots, v_{t} F_{t}, v_{t+1}=u$ where $F_{i}$ is a face of $P$ which contain the vertices $v_{i}$ and $v_{i+1}, i=1,2, \ldots, t$. Such a path is called a path of facets if $F_{i}$ is a facet of $P$ for every $i$. A path of faces is nested if, for every $i$, $\operatorname{dim} F_{i} \geq \operatorname{dim} F_{i+1}$ and if $\operatorname{dim} F_{i}>\operatorname{dim} F_{i+1}$, then $F_{i} \supset F_{j}$ for every $j>i$.

For two vertices $v$ and $w$ of $P$ let $I_{P}(u, v)$ denote a path of facets between $u$ and $v$ of minimal length. If $I_{P}(u, v)$ is of the form $u=v_{1}, F_{1}, v_{2}, F_{2}, \ldots, v_{t} F_{t}, v_{t+1}=u$, then it is easy to see that $N\left(F_{i}\right) \cap N\left(F_{j}\right)=\varnothing$ if $j \geq i+3$. Since otherwise if $F \in N\left(F_{i}\right) \cap N\left(F_{j}\right)$ we could make a shortcut by replacing the facets $F_{l}, i<l<j$, by $F$. (This is the crucial observation in Barnette's proof [1], [4].)

Proof of Theorem 1. Let $g(d, n)=(6 d)^{2 \log n} \leq n^{2 \log d+3}$. Note that $g(d, n)$ is a monotone function of $d$ and $n$, and that, for a fixed $d, g(d, n)$ is a convex function of $n$. We will prove by induction on $d$ that $\Delta(d, n) \leq g(d, n)$. This is clear for $d=1$. Assume that $\Delta\left(d^{\prime}, n\right) \leq g\left(d^{\prime}, n\right)$ for every $d^{\prime}<d$ and every $n$.

Let $P$ be a $d$-dimensional polyhedron with $n$ facets. Call a face $F$ of $P$ small if $|N(F)| \leq n / 2$, otherwise call $F$ big. Note that for every two big faces there is a facet which intersects them both.

Let $v$ and $u$ be two vertices of $P$. We will find two nested paths of faces $S_{1}$ and $S_{2}$ of the form

$$
\begin{array}{ll}
S_{1}: & v=v_{1}, F_{1}, v_{2}, F_{2}, \ldots, F_{s} \text { and } \\
S_{2}: & u=u_{1}, G_{1}, u_{2}, G_{2}, \ldots, G_{s},
\end{array}
$$


with the following properties:

(a) All faces $F_{i}, i \leq s$, and $G_{i}, i \leq t$, are small.

(b) There is a facet $F$ which intersects both $F_{s}$ and $G_{t}$.

(c) If $F_{i}$ and $F_{i+3}$ have the same dimension, then $N\left(F_{i}\right) \cap N\left(F_{i+3}\right)=\varnothing$, and the same property holds for the $G_{i}$ 's

Given $S_{1}$ and $S_{2}$, put $d_{i}=\operatorname{dim} F_{i}, d_{i}^{\prime}=\operatorname{dim} G_{i}, n_{i}=\left|N\left(F_{i}\right)\right|$, and $n_{i}^{\prime}=\left|N\left(G_{i}\right)\right|$. By the induction hypothesis, the distance between $v$ and $w$ in $G(P)$ is at most

$$
\sum_{i=1}^{s} g\left(d_{i}, n_{i}\right)+\Delta(d-1, n-1)+\sum_{i=1}^{t} g\left(d_{i}^{\prime}, n_{i}^{\prime}\right)
$$

Since $g(d, n)$ is a convex function of $n$ this expression is at most $\Delta(d-1, n-1)+$ $6 \sum_{i=1}^{d-1} g(d-i, n / 2)$.

It follows that $\Delta(d, n) \leq \Delta(d-1, n-1)+6 d g(d-1, n / 2)$ and therefore $\Delta(d, n) \leq 6 d^{2} g(d, n / 2)=g(d, n)$.

We will now describe the construction of $S_{1}$ and $S_{2}$. This will be done in at most $(d-1)^{2}$ steps which correspond to pairs of positive integers $(a, b), a$, $b \leq d-1$. In the $(a, b)$ th step we construct two nested paths of faces $R_{1}$ and $R_{2}$ :

$$
\begin{array}{ll}
R_{1}: & v=v_{1}, F_{1}, v_{2}, F_{2}, \ldots, F_{s} \text { and } \\
R_{2}: & u=u_{1}, G_{1}, u_{2}, G_{2}, \ldots, G_{t}
\end{array}
$$

with the properties:

(a') All faces $F_{i}, i<s$, and $G_{i}, i<t$, are small. $F_{s}$ and $G_{t}$ are big. $\operatorname{dim} F_{i}=d-a$ and $\operatorname{dim} G_{s}=d-b$.

(c') If $F_{i}$ and $F_{i+3}$ have the same dimension, then $N\left(F_{i}\right) \cap N\left(F_{i+3}\right)=\varnothing$, and the same holds for the $G_{i}$ 's.

To start the construction for $a=b=1$ consider $I=I_{p}(v, u)$. If there is only one big facet $F$ in $I$, break $I$ into two parts $S_{1}$ and $S_{2}$ by deleting $F$. If there is no big facet in $I$, break $I$ into $S_{1}$ and $S_{2}$ in an arbitrary way. Otherwise, let $R_{1}$ be the initial part of $I$ which ends with the first big facet of $I$, and let $R_{2}$ be (in reverse order) the terminal part of $I$ starting with the last big facet of $I$.

Assume that the sequences $R_{1}$ and $R_{2}$ are given. There is a facet $F$ which intersect both $F_{s}$ and $G_{t}$. (Since both are big faces.) Let $x$ be a vertex in $F_{s} \cap F$ and let $y$ be a vertex in $G_{t} \cap F$. Let $U=I_{F_{s}}\left(v_{s}, x\right)$ be a minimal path of facets of $F_{s}$ between the vertices $v_{s}$ and $x$ and let $W=I_{G}\left(y, u_{t}\right)$ be a minimal path of facets of $G_{t}$ between the vertices $y$ and $u_{t}$.

If all faces in the paths $U$ and $W$ are small construct $S_{1}$ and $S_{2}$ by replacing in $R_{1}$ the face $F_{s}$ by $U$ and by replacing in $R_{2}$ the face $G_{t}$ by $W$. Otherwise, assume that there is a big face in $U$ and let $H_{j}$ be the first big face in $U$. In this case, keep $R_{2}$ unchanged and replace in $R_{1}$ the face $F_{s}$ by the initial part of $U: v_{s}=a_{1}, H_{1}$, $a_{2}, H_{2}, \ldots, a_{j}, H_{j}$. (Note that $\operatorname{dim} H_{j}=d-a-1$.) It is immediate to check that these sequences satisfy the requirements for $(a+1, b)$.

Since every 1 -face is small this process will terminate with sequences $S_{1}$ and $S_{2}$ as required. 
Remarks. 1. Let $f(d, n)$ be a monotone function of $d$ and $n$ which is a convex function of $n$ for every fixed $d$. Suppose that $f(d, n)$ satisfies the relations $f(1, n) \geq 1$ and $f(d, n) \leq f(d-1, n-1)+\sum_{i=1}^{d-1} f(d-i, n / 2)$. The proof of Theorem 1 actually shows that $\Delta(d, n) \leq f(d, n)$.

For positive real numbers $a$ and $b$ define

$$
\begin{aligned}
& \bar{f}_{a, b}(d, n)=(a d / \log n)^{b \log n} \\
& f_{a, b}(d, n)=\max \left\{t \geq 1: t \cdot \bar{f}_{a, b}(d, n / t)\right\} .
\end{aligned}
$$

Note that $f_{a, b}(d, n)$ is a monotone function of $d$ and $n$ and is a convex function of $n$ for every fixed $d$.

The behavior of the function $f_{a, b}(d, n)$ is demonstrated by the following relations. There are constants $c>0$ and $C>1$ which depend on $a$ and $b$ such that $f_{a, b}(d, n) \leq \min \left\{n^{b \log d+c}, C^{d} \cdot n\right\}$. On the other hand, when $\log \log n=o(\log d)$ then $f_{a, b}(d, n) \geq n^{b \log d(1+o(1))}$ and when $\log n \geq c^{\prime \prime} \cdot d$ (for some constant $c^{\prime \prime}$ depending on $a$ and $b$ ) then $f_{a, b}(d, n) \geq C^{d} \cdot n$.

For $b=2$ and for $a$ sufficiently large $(a=20$ will do), the function $f(d, n)=$ $f_{a, b}(d, n)$ satisfies the relation

$$
f(d, n) \leq f(d-1, n)+\alpha f(d, \beta n),
$$

where $\alpha=\sqrt{6}$ and $\beta=\sqrt{2} / 2$. This relation (repeated twice) gives

$$
f(d, n) \leq f(d-1, n)+6 \sum_{i=0}^{d-1} f(d-i, n / 2) .
$$

It is clear that $f(1, n) \geq 1$. Thus, the proof of Theorem 1 gives

$$
\Delta(d, n) \leq f_{2,20}(d, n) .
$$

2. A substantial simplification of the proof giving a slightly better estimate, $\Delta(d, n) \leq n^{\log d+1}$, was recently obtained by Kleitman. See [6]. Kleitman's proof gives the recurrence relation $\Delta(d, n) \leq \Delta(d-1, n-1)+2 \Delta(d,\lfloor n / 2\rfloor)+2$.

\section{Subexponential Bounds for the Height}

We are given a $d$-dimensional polyhedron $P$ and a linear objective function $\varphi$. For simplicity we assume that $P$ is simple and that $\varphi$ is not constant on any edge of $P$. (Recall that a $d$-polyhedron $P$ is simple if every vertex of $P$ belongs to exactly $d$ facets.) There is no loss of generality in those assumptions. Let $r \geq 1$ be an integer. Given a vertex $v$ of $P$ consider the following algorithm $\mathrm{GI}(r)$ to reach the $\varphi$-maximal vertex. (GI stands for greatest improvement.)

GI $(r)$ : Start from a vertex $v$. If $v$ is not the $\varphi$-maximal vertex of $P$ replace $v$ by the vertex with maximal value of $\varphi$ among all vertices which belong to some $r$-face containing $v$, and repeat.

Note that GI(1) is just the simplex algorithm with Dantzig's original greatest improvement pivot rule. 
Theorem 4. If $P$ is given by $n \leq\lceil k d / 2\rceil$ linear inequalities in d variables, then for $r=d-\lceil d /(k-1)\rceil$ the algorithm $\mathrm{GI}(r)$ terminates after at most $2 k-5$ steps.

Lemma 5. Let $F_{1}, F_{2}, \ldots, F_{m}$ be $d$-subsets of the set $\{1,2, \ldots, n\}, n \leq\lceil k d / 2\rceil$. If $m \geq k-1$, then there are $i, j, 1 \leq i<j \leq m$, such that $\left|F_{i} \cap F_{j}\right| \geq\lceil d /(k-1)\rceil$.

Proof. Otherwise

$$
\left|\bigcup_{i=1}^{m} F_{i}\right| \geq(k-1) d-\left(\begin{array}{l}
k \\
2
\end{array}\right)(\lceil d /(k-1)\rceil-1)>\lceil k d / 2\rceil \geq n .
$$

Given a simple $d$-polyhedron $P$ let $G_{r}(P)$ denote the graph whose vertices are the vertices of $P$ and two vertices are adjacent if they are included in some $r$-face of $P$. (Thus $G_{1}(P)=G(P)$.)

Lemma 6. Let $P$ be a simple d-polyhedron with $n \leq\lceil k d / 2\rceil$ facets. Put $r=$ $d-\lceil d /(k-1)\rceil$. Then $G_{r}(P)$ does not contain an independent set of $k-1$ vertices.

Proof. To each vertex $v$ of $P$ associate the set $S(v)$ of facets containing $v$. Two vertices $w$ and $u$ belongs to some $r$-face iff $|S(w) \cap S(u)| \geq d-r$. Let $r=d-\lceil d /(k-1)\rceil$. Since there are altogether at most $\lceil d k / 2\rceil$ facets, Lemma 5 follows from Lemma 6.

Proof of Theorem 4. Let $\varphi$ be an objective function and let $v$ be the $\varphi$-maximal vertex of $P$. Let $w$ be a vertex of $P$, consider the sequence $w=w_{0}, w_{1}, \ldots, w_{m}=v$ where $w_{i+1}$ is the vertex with maximum value of $\varphi$ among all vertices which belong to some $r$-face containing $w_{i}$. If $m>2 k-5$, then $w_{0}, w_{2}, w_{4}, \ldots, w_{2 k-4}$ form an independent set of $k-1$ vertices in $G_{r}(P)$. By Lemma 6 this is impossible and therefore $m \leq 2 k-5$.

Proof of Theorem 2. From Theorem 4 it follows that if $n \leq\lceil k d / 2\rceil$, then

$$
H(d, n) \leq(2 k-5) H(d-\lceil d /(k-1)\rceil, n-\lceil d /(k-1)\rceil) .
$$

Note that if $(n / d) \leq(k / 2)$ for some integer $k \geq 2$, then

$$
(n-\Gamma d /(k-1)\rceil) /(d-\Gamma d /(k-1)\rceil) \leq(k+1) / 2
$$

Also note that $d-\lceil d /(k-1)\rceil=\lfloor d(k-2) /(k-1)\rfloor$.

By iterating (7) we obtain for every integer $t$ that

$$
H(d, n) \leq(2 k-5) \cdot(2 k-3) \cdot(2 k-1) \cdots(2 t-5)(2 t-3) H(x, y),
$$

where

$$
x=d\left\lceil\frac{k-2}{k-1} \cdot \frac{k-1}{k} \cdots \frac{t-1}{t}\right\rceil \leq\left\lceil\frac{(k-2) d}{t}\right\rceil
$$


and $y \leq(t+2) x / 2 \leq n$. Substituting $t=\sqrt{n}$ and using the trivial relation $H(d, n) \leq\left(\begin{array}{l}n \\ d\end{array}\right)$, we obtain that

$$
H(d, n) \leq(2 \sqrt{n}) !\left(\begin{array}{c}
n \\
2 \sqrt{n}
\end{array}\right) \leq n^{2 \sqrt{n}}
$$

Proof of Theorem 3. Let $h_{r}(d)$ be the maximum height of a polytope in $\mathscr{P}[d, r]$. It follows from Theorem 2 that $h_{r}(d) \leq(4 r-5) h_{r}(d-\lceil d /(2 r-1)\rceil)$. It follows that $h_{r}(d) \leq(4 r-5)^{\log (2 r-1) / 2 r-2 d d}=d^{C(r)}$ where $C(r)=\log _{(2 r-1) /(2 r-2)}(4 r-5)$. Note that $C(r)=O(r \log r)$. Let $P$ be a polytope in $\mathscr{P}[d, 2]$. Let $v$ be a vertex of $P$. There is a monotone path of length $h_{2}(d-1)$ from $v$ to a vertex $w$ with maximum $\varphi$ value among all vertices which belong to a facet containing $v$. If $w$ is not the $\varphi$-maximal vertex of $P$, then after moving along an improving edge from $v$ we must reach the only vertex of $P$ which belongs to no facet of $P$ containing $v$, and this vertex must be optimal.

Remarks. 1. Let $w(k, d, n)$ denote the maximal cardinality of an independent set in $G_{k}(P)$ over all $d$-dimensional polyhedra with $n$ facets. The value of $w(k, d, n)$ is closely related to the maximal possible number of binary vectors of length $n$, constant weight $d$, and Hamming distance at least $2 k+2$ apart. See Chapter 17 of [5]. In particular, Lemma 5 is a weak form of Johnson's bound for constant weight codes.

2. Lemma 6 implies that if $P$ is a $d$-polytope with at most $\lceil k d / 2\rceil$ facets, and $r=d-\lceil d /(k-1)\rceil$, then the diameter of $G_{r}(P)$ is at most $2 k-4$. By a similar argument we can prove that the diameter of $G_{r}(P)$ is actually at most $k-1$. The value $2 k-5$ in Theorem 4 can be reduced to $k-3$ by adding an improving move at the end of each step of algorithm $\mathrm{GI}(r)$.

3. In this remark we rely on the definitions in Section 1.5 of [3]. Let $\mathscr{G} \mathscr{F}$ denotes the class of polyhedra given as the set of generalized pseudoflows with a given excess function in some network (equipped with capacity and gain functions). It is easy to see that every face of a polyhedron in $\mathscr{G} \mathscr{F}$ is itself a polyhedron in $\mathscr{G} \mathscr{F}$. Another easy observation is that every polyhedron $P$ in $\mathscr{G}$ can be defined over a network with the property that all vertex degrees are at least 3 . If $P$ is defined over such a network with $v$ vertices and $e$ edges, then $d=\operatorname{dim} P=e-v$ and the number of facets of $P$ is at most $2 e \geq 6(e-v)=6 d$. It follows that $P \in \mathscr{P}[d, 6]$ and that the height of $P$ is at most $d^{\log _{(11 / 10)} 19}=d^{30.893 \ldots}$.

\section{General Combinatorial Setting}

\subsection{For the Diameter}

Let $K$ be a collection of $d$-sets. We call the elements of $\bigcup\{S: S \in K\}$ the vertices of $K$. Two sets in $K$ are adjacent if their intersection is of cardinality $d-1 . K$ is 
strongly connected if between every two sets $S$ and $T$ in $K$ there is a path of sets in $K$ of the form $S=R_{0}, R_{1}, \ldots, R_{t}=T$ such that, for every $i, R_{i}$ is adjacent to $R_{i+1}$. The distance between $S$ and $T$ is the minimum of $t$ over all such paths, and the diameter of $K$ is the maximal distance between two members of $K . K$ is ultraconnected if for every $S$ and $T$ in $K$ there is a path as above with the additional property that $(S \cap T) \subset R_{i}$ for every $i$.

Let $D(d, n)$ denote the maximal diameter of ultraconnected families of $d$-sets with $n$ vertices.

Clearly, $D(d, n) \geq \Delta(d, n)$. See, e.g., [7]. (It is known that any upper bound on the diameter of simple $d$-polyhedra with $n$ facets applies to general $d$-polyhedra with $n$ facets. Next, to each vertex $v$ of a simple $d$-polyhedron associate, as in the previous section, the set $S(v)$ of facets containing $v$. Two vertices $v$ and $w$ in $P$ are adjacent in $G(P)$ iff $S(v)$ is adjacent to $S(u)$. The family $\{S(v): v$ is a vertex of $P\}$ is ultraconnected since for every two vertices $u, v$ of $P$ which belong to some face $F$ of $P$ there is a path in $G(P)$ connecting $u$ and $v$ which lies in $F$.)

Larman's proof applies in this generality (and beyond it, see [10]) and gives $D(d, n) \leq 2^{d-1} n$.

The proof of Section 2 applies to $D(d, n)$. In fact, this is the context for which the proof was found. To see this let $K$ be an ultraconnected family of $d$-subsets of $[n]=\{1,2, \ldots, n\}$. Let $\bar{K}$ be the simplicial complex spanned by $K$, i.e., the set of all subsets of sets in $K$. For $S \in \bar{K}$ define $N(S)=\{i \in[n]:\{i\} \cup S \in \bar{K}\} . S$ is small if $|N(S)| \leq n / 2$ or if $|S|=d-1 . S$ is big otherwise. Now apply the proof of Section 2 word by word.

Kleitman's recent proof [6] can also be modified to apply to $D(d, n)$. To see this consider an ultraconnected family of $d$-subsets of $[m], m \geq n$, and a subset $V$ of $[m]$ of size $n$. Define the distance between two subsets of $V$ relative to $V$ to be minus one the minimal number of $d$-subsets of $V$ in a path in $K$ between $S$ and $T$. (In other words, $d$-sets which contain vertices not in $V$ are not counted.) The diameter of $K$ relative to $V$ is the maximum over all $S$ and $T$ of their distance relative to $V$. Let $\bar{D}(d, n)$ denote the maximal diameter of an ultraconnected family of sets relative to a set of $n$ vertices. Kleitman's argument applies in this context and gives the recurrence $\bar{D}(d, n) \leq \bar{D}(d-1, n-1)+2 \bar{D}(d, n / 2)+2$.

Remarks. 1. All our arguments apply to the more general (graph-theoretic) context considered by Larman [10].

2. A family $K$ of $d$-sets is ultraconnected iff the simplicial complex $\bar{K}$ is pure, and has the property that all links of faces of codimension one or more are connected. Note that this property is a topological property of $\vec{K}$.

\subsection{For the Height}

The proofs of the results on heights of polytopes apply directly to the following more general situation. Let $K=\left\{S_{1}, S_{2}, \ldots, S_{t}\right\}$ be a family of $d$-sets. For every $U$ let $i(U)$ be the maximal index so that $U \subset S_{i(U)}$. Suppose that, for every $U$, if $j<i(U)$ and $U \subset S_{j}$, then there is $l>j$ so that $U \subset S_{l}$ and $S_{l}$ is adjacent to $S_{j}$. 
In other words, every terminal subfamily of $K$ of the form $\left\{S_{j}, S_{j+1}, \ldots, S_{t}\right\}$ is ultraconnected. An "improving path" corresponds to a path of adjacent sets with increasing indices.

The required property for $K$ is much stronger than ultraconnectivity. It is equivalent to the assertion that the simplicial complex spanned by $K$ is shellable. Thus, the results on heights of polytopes apply to arbitrary shelling orders of the facets of a shellable complex.

\section{Final Remarks}

\subsection{What Is the Truth?}

The gap between the lower and upper bounds on $\Delta(d, n)$ is still substantial. The author's guess (which is as good as the reader's) is that the known upper bounds are asymptotically closer to the true value of $\Delta(d, n)$.

Conjecture 1. For some positive reals $a$ and $b$,

$$
D(d, n) \geq \Delta(d, n) \geq f_{a, b}(d, n) .
$$

(The function $f_{a, b}(d, n)$ is defined in Section 2.)

\subsection{Linear Programming}

There is a close relation between the complexity of edge-following algorithms for linear programming and the diameter problem for graphs of polytopes. Yet, good bounds for the diameter do not translate to quick pivot rules and, on the other hand, there are several transformations applicable to a linear program (such as LP duality) and it is possible that (allowing the use of these transformations) there is a worse-case polynomial variant of the simplex algorithm even if $\Delta(d, n)$ is not polynomial.

Klee and Minty [8] were the first to show that some variants of the simplex algorithm are exponential in the worse case. It would be of great interest to find a variant of the simplex algorithm for linear programming with subexponential worse-case behavior.

Consider the following algorithm $\mathrm{RI}(r)$.

$\mathrm{RI}(r):$ Start from a vertex $v$. If $v$ is not the $\varphi$-maximal vertex of $P$ choose a random $r$-face $F$ containing $v$, replace $v$ by the $\varphi$-maximal vertex in $F$ and repeat.

Conjecture 2. For every linear program with $d$ variables and $n \leq\lceil k d / 2\rceil$ inequalities, the expected number of iterations of $\operatorname{RI}(d-\lceil d /(k-1)\rceil$ is bounded by $O(k d \log n)$. 
This conjecture is obtained by trying to estimate the number of iterations needed, in a similar way to the proof of Theorem 4, using a generous amount of unjustified probabilistic independence assumptions.

If true, Conjecture 2 would give a subexponential (randomized) variant for the simplex algorithm. Moreover, we may apply this algorithm recursively and simultaneously for faces and their duals. (One of these will have no more facets than twice the dimension.) This shows that an affirmative solution to Conjecture 2 would imply a quasi-polynomial combinatorial randomized algorithm for linear programming.

Late Remark. Recently the author [12] found a subexponential variant of the simplex algorithm. Recursive applications of algorithm $\operatorname{RI}(r)$ for $r=d-1$ is subexponential but Conjecture 2 remains open.

\section{Acknowledgments}

I am thankful to Jeff Kahn and Nimrod Megiddo for fruitful discussions.

\section{References}

1. D. W. Barnette, $W_{v}$ paths on 3-polytopes, J. Combin. Theory 7 (1969), 62-70.

2. D. B. Dantzig, Linear Programming and Extensions, Princeton University Press, Princeton, NJ, 1963.

3. A. V. Goldberg, E. Tardos, and R. E. Tarjan, Network flow algorithms, in Path, Flows and VLSI Layout, (B. Korte et al., eds.), Springer-Verlag, New York, 1990, pp. 101-164.

4. B. Grünbaum, Convex Polytopes, Wiley Interscience, London, 1967, Chapter 16.

5. G. Kalai, The diameter problem for convex polytopes and $f$-vector theory, in The Victor Klee Festschrift (P. Gritzman and B. Strumfels, eds.), American Mathematical Society, Providence, RI, 1991, pp. $387-411$.

6. G. Kalai and D. J. Kleitman, A quasi-polynomial bound for diameter of graphs of polyhedra, Research Announcement, Bull. Amer. Math. Soc. 24 (1992), in press.

7. V. Klee and P. Kleinschmidt, The $d$-steps conjecture and its relatives, Math. Oper. Res. 12 (1987), $718-755$.

8. V. Klee and G. J. Minty, How good is the simplex algorithm? in Inequalities, Vol. III (O. Shisha, ed.), Academic Press, New York, 1972, pp. 159-175.

9. V. Klee and D. Walkup, The $d$-step conjecture for polyhedra of dimension $d<6$, Acta Math. 133 (1967), 53-78.

10. D. G. Larman, Paths on polytopes, Proc. London Math. Soc. 20 (1970), 161-178.

11. P. McMullen, The maximal number of faces of a convex polytope, Mathematika 17 (1970), 179-184.

12. G. Kalai, A subexponential randomized simplex algorithm, Proc. 24th ACM Symposium on Theory of Computing, 1992, to appear.

Received January 28, 1991, and in revised form August 26, 1991, and November 16, 1991. 